\title{
Solitary necrotic nodule of the liver
}

INSERM

\section{Source}

INSERM. (1999). Orphanet: an online rare disease and orphan drug data base. Solitary necrotic nodule of the liver. ORPHA:100035

Solitary necrotic tumor of the liver is a rare nonmalignant hepatic lesion characterized by a mass with a completely necrotic core often partially calcified, surrounded by a dense hyalinized fibrous capsule containing elastin fibers. Patients are usually asymptomatic but some may suffer from intermittent abdominal pain or discomfort. 